\title{
Development of an AR system for the advancement of the tasks in the construction sites
}

\author{
Bikash Lamsal $^{\text {a* }}$, Kyosuke Kunichika ${ }^{a}$, \\ ${ }^{a}$ Kajima Technical Research Institute, Kajima Corporation 182-0036 Tobitakyu, Chofu, Tokyo, Japan, lamsal@kajima.com
}

\begin{abstract}
In this paper, we developed an AR (Augmented Reality) application for productivity improvement in the construction sites. We developed this system for upgrading the ancient methods of construction by using the AR Technology. The main concept of this system is to use the AR technology for visualizing the completed form of the buildings, reforming the "construction management" and improving the productivity on the construction sites. This system can be used for the "presentation of the completed models" for early decision of the task to be performed, "safety work confirmation" for eliminating and reducing the dangerous tasks, "visualization of completed model before the completion of the buildings" for eradication of serious mistakes on quality of the buildings to be build. Generally, the AR system is used along with various types of head worn devices like Head mount displays or Google glass, but they are not realistic for the construction workers or in the construction sites. Our application works on iPad ${ }^{\circledR}{ }^{\circledR}$ and tablets which is easy to use and is a realistic form of using AR in the construction sites. Our application is developed in such a way that, the AR model generated from the BIM (Building Information Modeling), projected on 1:1 scale in a real space is continuously displayed in a smart device following the movement of the person holding the tablet. As a person moves holding the tablet, the position and orientation of the tablet is tracked, and the model follows the tablet. The developed system is experimented on the building construction sites in Japan. The experiments were conducted by developing the AR models from the BIM model of the various areas of the architectural construction sites. The experimental results reflect the effectivity of our AR system for increasing the productivity of the construction sites.
\end{abstract}

(C) 2019 The Authors. Published by Budapest University of Technology and Economics \& Diamond Congress Ltd.

Peer-review under responsibility of the scientific committee of the Creative Construction Conference 2019.

Keywords: Architecture; Augmented Reality; Building Information Modeling (BIM); Construction;

\section{Introduction}

Augmented Reality (AR) is a Computer vision system that gives a view of the real world where the elements are superimposed by computer generated files as graphics, sounds, videos or digital information. AR was first developed in 1968 by Ivan Sutherland at Harvard, where the users were able to experience the computer graphics that made them feel being in the alternate reality. Been around for numerous decades, various developments have been made in the field of AR. The implementation of AR in the medical and various other fields are spreading as a part of business innovation but AR technologies were not applied on construction sites due to the tracking and alignment problems in the construction sites, instead they were used at the office for simulation or collaboration during the design phase of a project.

In the recent years, a huge development in the field of AR technology have been made, which shows the possibilities of applying the AR in the field of construction sites. The construction industry is dependent on visual imaging solutions for achieving the functions related and performance related information. One of the leading 
innovations for visual imaging in the field of ICT Augmented Reality (AR), which brings virtual objects in the same axis with real world and enables monitoring the virtual objects together with the real world where they are relayed (1). Shin and Dunston (2) states that the AR technology provides various advantages through visualization and Simulation of the construction industry. As an example, allowing the observer to interact with both the actual and the virtual objects and monitor the construction progress by comparing the as planned and as built status of the project (2). In the past research, Wang and Dunston mentioned that it is very impracticable to train a heavy construction equipment operator in a real condition, so they developed an AR based heavy equipment operator training system called ARTS (3). A 4-dimensional AR model was developed for automating the construction progress monitoring and data collection, processing and communication in the construction phase of the project (4). Various web based augmented panoramic environment are developed for the documentation of the construction progress (5).

The common problems like, delay and misrecognition of the fixed things, critical quality accident of the frame (pile, rebar etc.), mistakes in finishing materials, misplacing equipment's, inadequate provisional equipment's, lack of building maintenance information and others still occur in the construction sites. These problems are time consuming as well expensive. To overcome with these problems one by one, we think of some ideas on using the AR in the field of constructions to bring the advancement in the ancient tasks performed in the construction sites. We develop a concept of mobile AR application, aimed to visualize the completed form of the models in the construction sites. Since, we have developed an AR application that can be used on the iPad ${ }^{\circledR} \mathrm{s}$ on the construction sites. In this paper, we will introduce about this application and its real implementation in the construction fields by on-site employees.

Our application is designed to be used in an $\mathrm{iPad}{ }^{\circledR}$ by connecting with the structure sensor. The two fiducial markers are installed at the local reference point in the sites for detecting the position of the model to be displayed in the $\mathrm{iPad} \otimes$ screen. When the two fiducial markers are detected by the structure sensors, the AR model generated form the BIM (Building Information Modelling), projected on 1:1 scale in a real space is continuously displayed in an iPad® following the movement of the person holding the iPad $\AA$. This application was experimented and tested in various construction sites in Japan for validating the effectiveness of our AR application.

\section{Related Works}

The mobile AR application was first presented in the Columbia University Campus for viewing the information around the campus area, with a PC and Head Mount Display (HMD) (6). After some years, the marker-based AR system (7) was introduced. The system was then developed by combining the hybrid computer vision and sensor-based tracking for mobile outdoor applications (8). AR tracking these days are available for mobile phones and tablets being based on the GPS and compass sensors or IMU device. An accurate 3D tracking technology in the construction sites was introduced by utilizing the 3D points clouds generated from the site photos (9). The tracking system developed by using the 3D point clouds were then made able to work on live videos (10).

Due to the limitations of the GPS, the mobile AR application are being used widely in the outdoor environment. In case of the indoor environment, the localization of the AR points or the device is a challenging job. A mobile AR application presented few years ago combine various computer vision tools, $3 \mathrm{D}$ reconstructions and sensors to provide reliable tracking (11), This system integrate the BIM data in their system. Our application is somewhat similar with these system in the current perspective. The Info SPOT system was an approach for mobile AR maintenance that utilized BIM for facility management based on predefined natural markers (12). Various related works have been done, but the difference to our system is, we are using the extended AR tracking system and a location-based AR model visualization process. We are using the structure device for calculating the movement and the position of the device, the fiducial markers are used to localize the point for displaying the AR model.

\section{AR Application Prototype}

\subsection{Overview}

The tasks performed in the construction sites are generally done by using the traditional methods and are the best way for getting the proper output, but it depends on the construction workers and their experiences. In the coming future, the Japanese Constructions will lack a lot of manpower to work in the sites because of the decreasing population in Japan, the old and experienced people will be retired, and the young peoples are not interested to work in the construction sites. Apart from this, a lot of problems related to the tasks in the construction sites usually occurs in a huge amount almost every day. The problems can be classified as the "delay and misunderstanding in fixing and decision making", "Serious quality accident of the frame (Pile, rebar etc.)", "facility positioning error", "mistake in the finishing materials", "Inadequate temporary facilities", "lack of building maintenance information" and so on. To overcome with these problems, we developed an AR application for the advancement of the tasks in the construction 
Bikash Lamsal, et al./ Proceedings of the Creative Construction Conference (2019) 114 https://doi.org/10.3311/CCC2019-114

sites. The current application is designed and developed to use in the construction sites by using the smart devices such as $i \mathrm{Pad}^{\mathbb{R}}$, iPhone $^{\mathbb{R}}$, tablets ${ }^{\mathbb{R}}$ etc. The main concept of our current application is to visualize the completed form of the BIM models in the construction sites using the AR technology.

\subsection{Structure of the Proposed algorithm}

The structure of our proposed algorithm is shown in figure 1. We have divided the structure of our algorithms in two parts, first part is the preprocessing as shown in figure 1(a) and second part is the main process of the algorithm as shown in figure 1 (b). In the pre-processing of our proposed algorithm, we work on developing the AR models from the BIM model and fixing the positional relationship of the two AR marker points. We have used the Vuforia ${ }^{\mathrm{TM}}$ TM (13) for fixing the positional relationship of the two AR marker points and save them in the device. The positional relationship of the two AR marker points will be effective for detecting the position of the smart device and continuous display of the AR model with high accuracy in the real construction sites.

In the main process of our proposed algorithm, we attach an external structure sensor (14) with the smart device. As the application is turned on, the structure sensor attached with the $\mathrm{iPad} \otimes$ is also turned on. When the structure sensor is turned on, AR space is constructed by using the IMU device, gyro sensor, camera image and depth camera image from the device. The two markers are then placed in the local reference point fixed during the pre-processing. The two markers are then detected by using the infrared camera of the structure sensor. Then the system sets the AR space for displaying the AR model based on the co-ordinates of stored AR marker (two points). AR function is then implemented by superimposing the image capturing the real space and the image capturing the AR space, which in results displays the AR model in the $\mathrm{iPad} \circledast$ screen with the scale of 1:1. The AR model is displayed continuously even after the loss of the marker. The tracking algorithm of the structure sensor SDK (14) is used for tracking the position of the device and reflects it in the AR space even the device moves or changes its position. The workers can inspect their works in the construction sites in a real time by comparing the real image and the AR transformed BIM model. The pictorial representation of our proposed algorithm is shown in figure 2 .

a

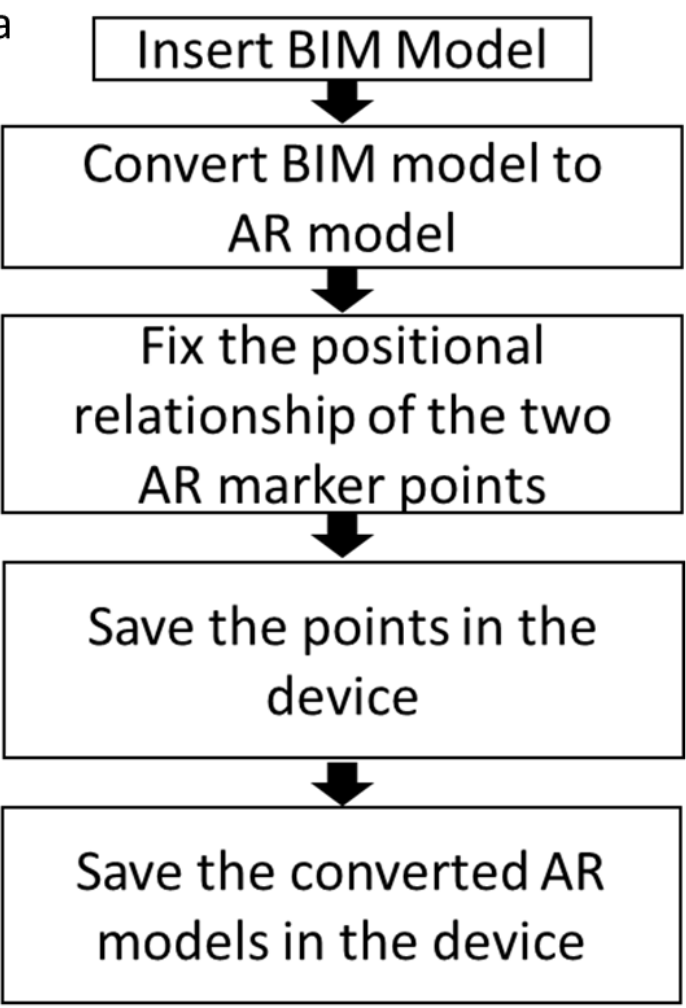

$\mathrm{b}$

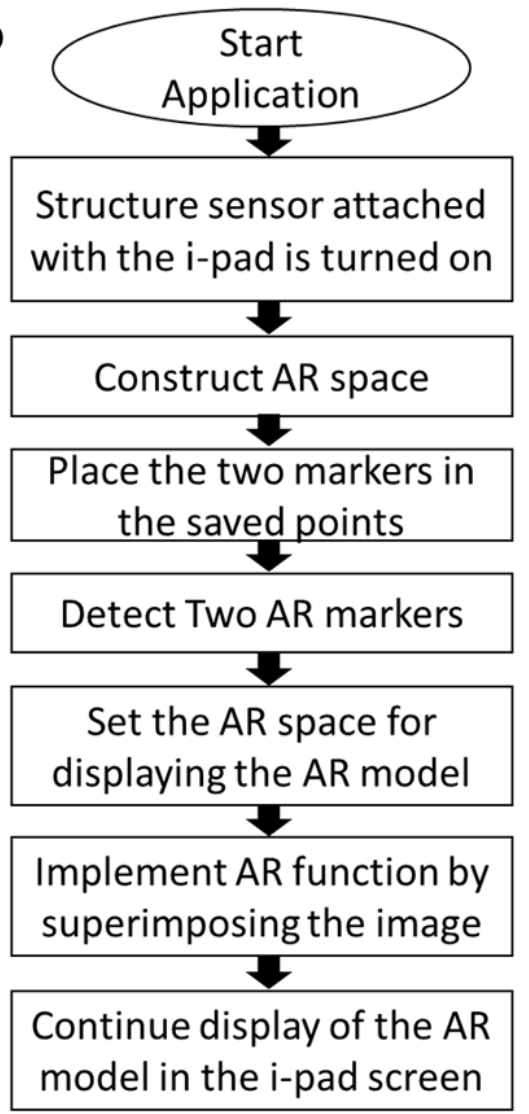

Fig. 1. Proposed algorithm: (a) Pre-processing of the proposed algorithm, (b) Main Process 
Bikash Lamsal, et al./ Proceedings of the Creative Construction Conference (2019) 114 https://doi.org/10.3311/CCC2019-114
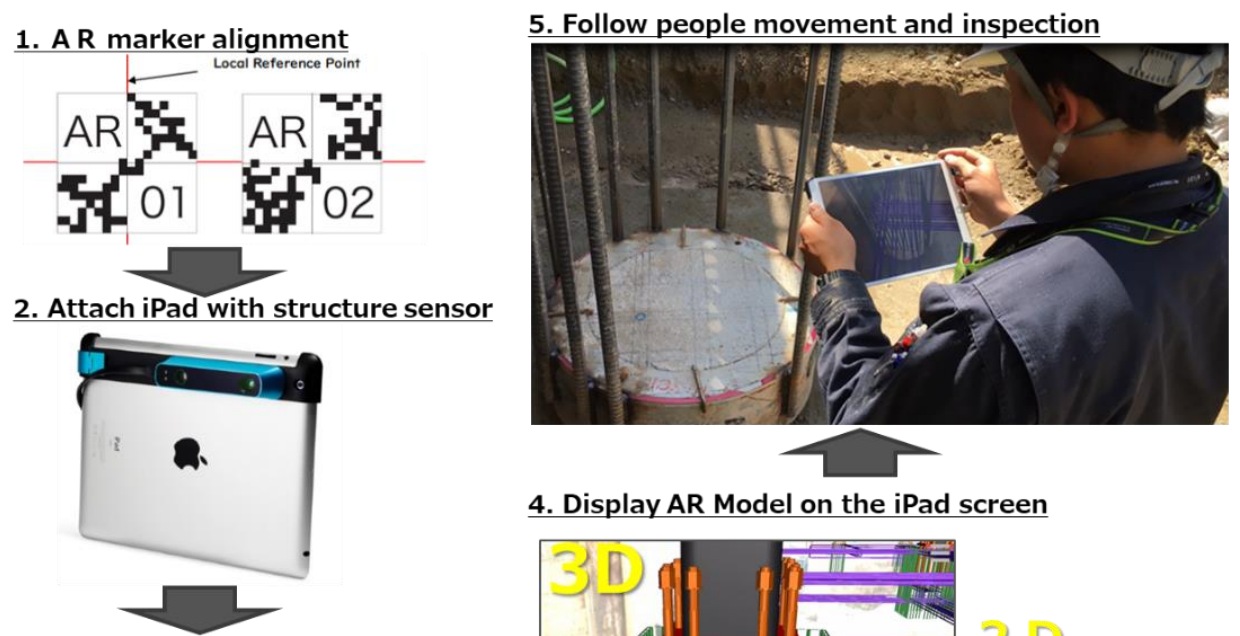

4. Display AR Model on the iPad screen

3. AR marker detection
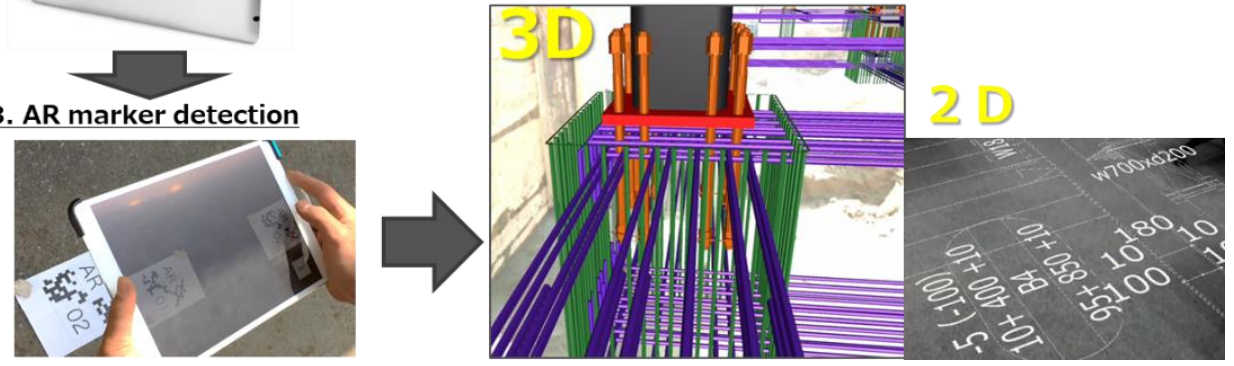

Fig.2. Pictorial representation of our proposed algorithm

\subsection{Test case}

We choose a 13-storey steel building site for testing our current AR application. The application was applied for the task related to basic reinforcement of the Rebar construction. This task is one of the complicated and complex tasks, where the mistakes and errors related to the foundation beam and foundation pillar occurs very easily. To avoid such mistakes, we developed several AR models from the BIM models for getting the prior confirmation of the completed model in the real sites before starting the tasks or in the middle of the tasks or whenever the workers gets confused. This process is very much effective for reducing the mistakes and errors in the construction sites. In figure 3 , image (a) displays the site before applying the AR processing, image (b) displays the 3D image of the site after applying the $\mathrm{AR}$ processing and image (c) displays the $2 \mathrm{D}$ image of the site after applying $\mathrm{AR}$ processing.

a

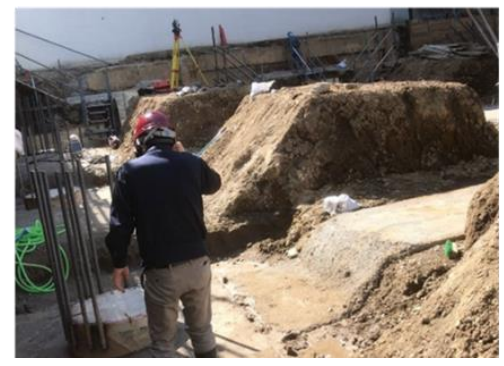

b

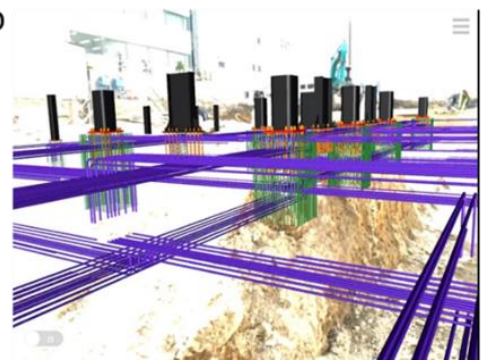

c

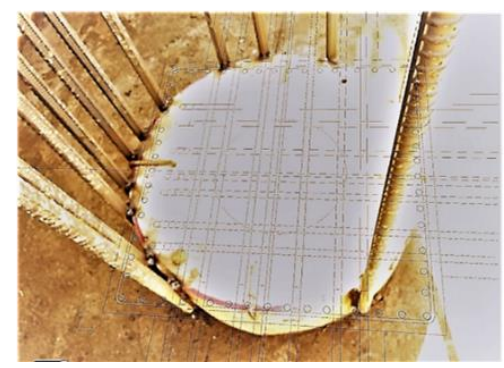

Fig.3 (a). Site before Rebar construction (b). 3D model of Rebar construction (c). 2D model of Rebar construction

\subsection{AR Models}

In this paper, we focused on the Rebar construction and the Rebar construction is combined of various stages including various parts. In this application we developed and implemented the models related to the Rebar construction. The 3D model of bottom reinforcement, top reinforcement, Pillar and combined model of top and bottom beam reinforcement models were developed for testing in the construction sites. The models are shown in the figure 4 . These models are developed for confirming the model before building and eliminate the mistakes and error. 

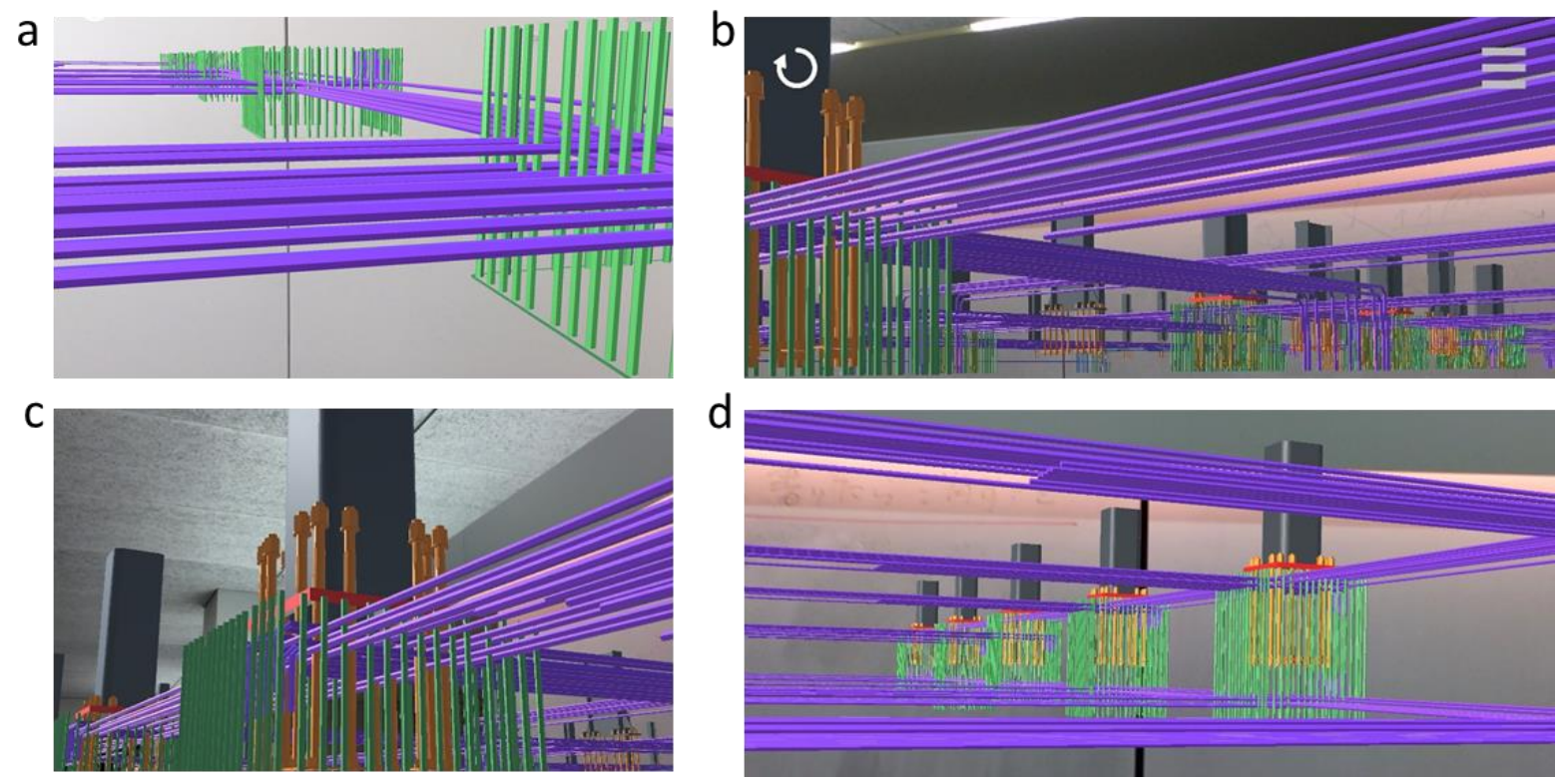

Fig.4 (a). 3D model for bottom reinforcement (b). 3D model for top reinforcement (c). 3D model for pillar (d). 3D model of combined top and bottom reinforcement

\section{Testing and Evaluation}

We tested our application in the 13-storey steel building construction site of Japan. The test was performed by the construction workers from the main contractor and the workers from the Rebar company for confirming the 3D and 2D models of the Rebar before constructing the basic reinforcement in the site. The application was tested by placing the two AR markers in the site and localized the position of the iPad ${ }^{\circledR}$ by using our application which results in the display of the 3D reinforcement models in the iPad ${ }^{\circledR}$ screen in the scale of 1:1. The model follows the movement of the device and display the model in the proper location calculated from the marker points using the IMU device, Gyro sensor, Real image space and the AR image space.

After testing the application, the site's member evaluated our application as an useful tools in the construction sites, this application can be used in the construction sites as a tool to show the completed model to the customer in the open land, can be used as an educational material because the models are easy to see and understand as they are seen locally in the sites and also can be used for the interference check during the temporary design. Similarly, the Rebar company also evaluated our application as an effective tool for understanding the complex layout model of the basic reinforcement on site in a real time. Our application will be helpful for the prevention of re-arrangement, can be used for checking the joint bar and can be used for checking the reverse strike joint bar for inverted construction method. Due to the problems related to the handshake, IMU device and other hardware tools, the error related to the positioning and localization of the device occurs.

\section{Conclusion}

In this paper, we have described about the development and the implementation of a prototype AR application to be used in the construction sites. Based on the testing and the evaluation of our application by the construction workers, the application seems to be effective for the construction workers to confirm the complete image of the building models and the materials to be used, the application helped to create the image of the completed model and displayed the model on the local sites with the proper localization of the device in a real time. The iPad ${ }^{\circledR}$ was used as an AR tool for displaying the AR model, is one of the best ideas to be applied in the construction sites in comparison to the head worn devices and the glasses. The use of two markers seemed to be time consuming and needs to be reduced to one marker or marker less. The localization of the device suffered an error in $\mathrm{cm}$ precision needs to be improved for using this application in the various construction related tasks. In this paper, we tested with the rebar construction models, but the application can be used for any other models for prior confirmation and visualization of the construction sites locally. 
Bikash Lamsal, et al./ Proceedings of the Creative Construction Conference (2019) 114

https://doi.org/10.3311/CCC2019-114

\section{References}

[1] Hammad, A., Wang H., \& Mudur S. P., Distributed augmented reality for visualizing collaborative construction tasks. Journal of Computing in Civil Engineering, (2009), Vol.23, No. 6. http://dx.doi.org/10.1061/(ASCE)0887-3801(2009)23:6(418)

[2] Shin, DH, \& Dunston, PS., Identification of application areas for Augmented reality in industrial construction based on technology suitability. Journal of Automation in Construction, Elsevier, 17 (2008), 882-894. https://doi.org/10.1016/j.autcon.2008.02.012

[3] Wang X., \& Dunston P.S., (2007). Design, strategies, and issues towards an augmented reality-based construction training platform, ITcon, 12 (2007), 363-380.

[4] Golparvar-Fard, M, Peña-Mora, F, \& Savarese, S., D4AR - A 4-dimensional augmented reality model for automating construction progress monitoring, data collection, processing and communication. Journal of Information Technology in Construction, ITcon, 14 (2009), $129-153$.

[5] Waugh, LM, Rausch, B, Engram, T, \& Aziz, F., Inuvik Super School VR Documentation: Mid-Project Status. Quebec, Canada: CSCE, 15 ${ }^{\text {th }}$ International Conference on Cold Regions Engineering, (2012). https://doi.org/10.1061/9780784412473.022

[6] Feiner S., MacIntyre B., Höllerer T., Webster A. A touring machine: prototyping 3D mobile augmented reality systems for exploring the urban environment. In Proc. ISWC'97, Cambridge, MA, USA, Oct 13, 1997. https://doi.org/10.1109/ISWC.1997.629922

[7] Pasman W. and Woodward C., Implementation of an augmented reality system on a PDA", Proc. ISMAR2003, Tokyo, Japan, Oct 2003, pp. 276-277. https://doi.org/10.1109/ISMAR.2003.1240718

[8] Reitmayr G. and Drummond T., Going out: robust, model-based tracking for outdoor augmented reality, Proc. ISMAR2006, Santa Barbara, USA, 22-25 Oct 2006, pp.109-118. https://doi.org/10.1109/ISMAR.2006.297801

[9] Peña-Mora F., Golparvar-Fard M., Fukuchi Y., Savarese S., D4AR 4-Dimensional Augmented Reality models for automation and interactive visualization of construction progress monitoring. Proc. CONVR2010, Sendai, Japan, Nov 4-5, 2010, pp. 15-24.

[10] C. Woodward, T. Kuula, P. Honkamaa, M. Hakkarainen, P. Kemppi, Implementation and evaluation of a mobile augmented reality system for building maintenance, Proceedings of the $14^{\text {th }}$ Intl. Conference on Construction Applications of Virtual Reality, Sharjah, 2014.

[11] Kahn S., Olbric M.h, Engelke T.,Keil J.,Riess P., Webel S., Graf H., Bockholt U., Beyond 3D "as-built" information using mobile AR enhancing the building lifecycle management, Proc. Intl. Conference on Cyberworlds, Darmstadt, Germany, (2012), pp. 29-36. https://doi.org/10.1109/CW.2012.12

[12] Irizarry J., Gheisari M., Williams G., Walker B.N., Info SPOT: A mobile augmented reality method for accessing building information through a situation awareness approach, Automation in Construction 33 (2013), pp. 1123 https://doi.org/10.1016/j.autcon.2012.09.002

[13] Kincade K.W., Cityscape: An Augmented Reality Application for Displaying 3D Maps on a Mobile Device, Colorado School Of Mines, (2014), pp. 1-7.

[14] "Structure Sensor" in , Inc. Occipital, [online] Available: http://structure.io/. 